\title{
Estrategia didáctica mediada por el aprendizaje autorregulado para el desarrollo del pensamiento crítico en educación artística
}

\section{Didactic strategy mediated by self-regulated learning for the development of critical thinking in arts education}

Sandra Milena Puerta-Vásquez

Corporación Universitaria Minuto de Dios, Medellín, Colombia

sandra.puerta@uniminuto.edu.co

https://orcid.org/0000-0002-8238-4815

Verónica Johana Suárez-Molina

Corporación Universitaria Minuto de Dios, Bogotá, Colombia

vsuarezmoli@uniminuto.edu.co

https://orcid.org/0000-0002-1422-4959

Recepción: 20/10/2021 | Aceptación: 06/01/2022 | Publicación: 10/01/2022

Cómo citar (APA, séptima edición):

Puerta-Vásquez, S. M., y Suárez-Molina, V. J. (2022). Estrategia didáctica mediada por el aprendizaje autorregulado para el desarrollo del pensamiento crítico en educación artística. INNOVA Research Journal, 7(1), 38-58. https://doi.org/10.33890/innova.v7.n1.2022.1979

\section{Resumen}

Esta investigación tuvo como propósito diseñar una estrategia didáctica mediada por el aprendizaje autorregulado para el desarrollo de habilidades del pensamiento crítico en el área de educación artística en la disciplina de artes plásticas y visuales. Partiendo de un enfoque mixto, se inició, desde un estudio cuantitativo a través de un diseño no experimental, con la identificación de las habilidades del pensamiento crítico y las competencias de autorregulación de los estudiantes. Luego, se dio desarrollo al estudio cualitativo, en el que, por medio de un diseño fenomenológico, se determinaron los componentes de la estrategia didáctica y se analizaron los factores de la autorregulación del aprendizaje en esta. Para la recolección de los datos cuantitativos se aplicó un cuestionario a 27 estudiante, mientras que para la obtención de los datos cualitativos se realizó una entrevista semiestructurada a 5 docentes. Entonces, con base en un análisis descriptivo, se evidenció que, si bien existen habilidades del pensamiento crítico y competencias de autorregulación en los estudiantes, estas no están desarrolladas en gran medida y requieren de un 
Estrategia didáctica mediada por el aprendizaje autorregulado para el desarrollo del pensamiento crítico en educación artística

fortalecimiento. Además, se señaló que para el desarrollo de la estrategia didáctica los docentes proponen plantear actividades artísticas que contengan elementos motivadores y de autorreflexión. Así, es de gran importancia el rescate que se realizó en esta investigación a las virtudes del arte y el cómo a través de la autorregulación se desarrollan habilidades del pensamiento crítico, lo que puede llevar a la transformación personal y social de los jóvenes.

Palabras claves: estrategia didáctica; aprendizaje autorregulado; pensamiento crítico; educación artística; educación media.

\begin{abstract}
The purpose of this research was to design a didactic strategy mediated by self-regulated learning for the development of critical thinking skills in the area of artistic education in the discipline of plastic and visual arts. Starting from a mixed approach, it began, from a quantitative study through a non-experimental design, with the identification of students' critical thinking skills and selfregulation skills. Then, the qualitative study was developed, in which, through a phenomenological design, the components of the didactic strategy were determined and the factors of the selfregulation of learning in it were analyzed. To collect quantitative data, a self-administered questionnaire was applied to 27 students, while to obtain qualitative data, a semi-structured interview was carried out with 5 teachers. Then, based on a descriptive analysis, it was evidenced that, although there are critical thinking skills and self-regulation skills in students, these are not developed to a great extent and require strengthening. In addition, it was pointed out that for the development of the didactic strategy the teachers suggest to propose artistic activities that contain motivating elements and self-reflection. Thus, the rescue that was carried out in this research to the virtues of art and how through self-regulation critical thinking skills are developed, which can lead to personal and social transformation of young people has a great importance.

Keywords: didactic strategy; self-regulated learning; critical thinking; artistic education; middle education.
\end{abstract}

\title{
Introducción
}

A través de la historia, el arte ha servido como vehículo para los procesos de comunicación entre los seres humanos, y a su vez, como un catalizador para la transformación social (Tolosa, 2015). Es así como, la obra de arte es una creación que surge a partir de la experiencia estética del artista; de lo que siente y piensa sobre lo que sucede en su entorno y en ese sentido, en muchas ocasiones se convierte en una crítica acerca del contexto social inmediato, es decir; en una herramienta para el desarrollo del pensamiento crítico (Huertas, 2010).

A pesar de lo anterior, el área de educación artística en el contexto escolar colombiano ha sido históricamente enfocada, de manera errada, hacia el desarrollo de actividades manuales o expresivas que carecen de un vínculo con el ejercicio intelectual, el cual puede ayudar en gran medida al desarrollo personal y social de los estudiantes. Así que, con el fin de reivindicar el verdadero propósito educativo del área, en este estudio señala la importancia de la práctica artística en el desarrollo de procesos cognitivos como el pensamiento crítico y el aprendizaje autorregulado. Como lo resalta Álvarez (2018) en su estudio "La autorregulación en el aprendizaje del estudiante durante el proceso artístico de la creación consciente", las vivencias socioculturales que surgen en la práctica artística, en las que el estudiante juega, interactúa, interpreta y crea, facilitan la 
autorregulación de su aprendizaje y el desarrollo del pensamiento crítico hacia sus diferentes realidades.

Por otro lado, Vallejo (2017) analizó la influencia de la autorregulación del aprendizaje en la disciplina escolar de los estudiantes de bachillerato, encontrando que si la autorregulación no es fortalecida en los estudiantes se pueden presentar dificultades en el desarrollo del pensamiento y el control de las emociones, lo que incrementa el bajo rendimiento escolar y contribuye a la generación de impulsos negativos que originan comportamientos inadecuados. Entonces, teniendo en cuenta lo planteado por el Ministerio de Educación Nacional (2010), la educación artística, partiendo de procesos como el de la sensibilización, puede contribuir al desarrollo de competencias que le permitan al estudiante el conocimiento y la identificación de las emociones propias y ajenas, lo que disminuye las dificultades en cuanto al autocontrol emocional que resultan de la carencia de autorregulación en su aprendizaje.

Así, se reconoce la importancia de que el estudiante se forme como persona autónoma y social y para ello, deben facilitarse espacios de aprendizaje donde este desarrolle competencias que le permitan afrontar las diferentes situaciones que se presentan en su cotidianidad y, entonces, pueda desenvolverse de manera asertiva en la sociedad (García, 2005). En este sentido, tiene cabida la autorregulación del aprendizaje como medio para el desarrollo de habilidades de pensamiento crítico en tanto permite al estudiante el éxito de los procesos de formación social y académica, ya que el alumno puede concentrar esfuerzos y dirigir sus objetivos hacia una meta propuesta, demostrando que la autonomía favorece la capacidad de aprender.

De esta forma, como docentes es vital tener información que permita analizar la incidencia de la autorregulación del aprendizaje en el fortalecimiento de las habilidades del pensamiento crítico dentro del área de educación artística como inicio para aportar al aprendizaje efectivo en el aula de clase y a la proyección que el estudiante tendrá a futuro como ciudadano, por lo que esta investigación aporta a los docentes insumos y temáticas relacionadas con el aprendizaje autorregulado desde las estrategias que pueden servir para la promoción y desarrollo de habilidades de pensamiento crítico en el área de artística, esperando que sirva como texto de consulta para futuras investigaciones referentes a la misma temática y fortalezca el conocimiento, las prácticas educativas.

En este sentido, con relación al contexto educativo en el que se enmarca la investigación, la cual se centra en estudiantes de básica media, es importante señalar que en el aspecto social existen varios factores de riego que sitúan a los estudiantes en una condición de vulnerabilidad, lo que contribuye a la existencia de un bajo rendimiento académico, una falta de interés por su proceso de aprendizaje y numerosos casos de deserción y pérdida del año. Por lo anterior, es necesario implementar la autorregulación como medio para el desarrollo del pensamiento crítico en los estudiantes, pues de esta forma influirá en el fortalecimiento de habilidades que les facilite el ejercicio de un análisis contextual con el que podrán identificar los obstáculos presentes en su cotidianidad que impiden su pleno desarrollo; además, podrán encontrar soluciones que lleven a la mitigación de los mismos (Vicuña y Sanjinés, 2018). A su vez, podrán dar solución a muchas de las actividades planteadas en las diferentes áreas del conocimiento, pues el pensamiento crítico y la autorregulación son competencias que le permiten al estudiante desarrollar una lectura textual, espacial y simbólica (García, 2005), por lo que se convierte en la capacidad para resolver variadas 
Estrategia didáctica mediada por el aprendizaje autorregulado para el desarrollo del pensamiento crítico en educación artística

actividades educativas, como solucionar problemas matemáticos, realizar análisis de casos, construir ensayos, exponer y defender sus ideas en un debate, entre otras.

Partiendo de lo anterior, y con base en lo consignado dentro de las comisiones de promoción de la institución educativa, se identificaron como determinantes del problema de estudio los siguientes aspectos: en primer lugar, el poco interés por parte de estos estudiantes en su proceso formativo, académico y personal, lo cual puede estar relacionado con el contexto social anteriormente mencionado y con la ausencia de competencias en cuanto al aprendizaje autorregulado. Pues, según Vicuña y Sanjinés (2018) un estudiante que autorregula su aprendizaje debe mantenerse en constante automotivación y por ende desarrollar su sentido de la autoeficacia. En segundo lugar, el bajo rendimiento académico que existe en este grado, especialmente en las áreas de matemáticas, lengua castellana, ciencias naturales y educación artística. Finalmente, la desestimación que se tiene en general del área de educación artística. Pues, a pesar de las orientaciones pedagógicas planteadas por el Ministerio de Educación Nacional en (2010), las cuales señalan la relación existente entre las competencias artísticas y las competencias básicas en comunicación, matemáticas, ciencias y ciudadanía; en el contexto escolar colombiano esta ha sido históricamente enfocada, de manera errada, hacia el desarrollo de actividades manuales o expresivas que carecen de un vínculo con el ejercicio intelectual y el desarrollo del pensamiento crítico, el cual, según huertas (2010) tiene relación directa con la utilidad social del arte y debe ser la base de la enseñanza dentro del área.

Por consiguiente, se planteó como pregunta general de investigación: ¿qué estrategia didáctica mediada por el aprendizaje autorregulado posibilita el desarrollo de habilidades del pensamiento crítico en el área de educación artística en la disciplina de artes plásticas y visuales? Entonces, partiendo de esta pregunta, se estableció como objetivo general del estudio: diseñar una estrategia didáctica mediada por el aprendizaje autorregulado para el desarrollo de habilidades del pensamiento crítico en el área de educación artística en la disciplina de artes plásticas y visuales. A su vez, se definieron como objetivos específicos: 1) identificar las habilidades de pensamiento crítico y las competencias de autorregulación que tienen los estudiantes, 2) determinar los componentes de la estrategia didáctica mediada por el aprendizaje autorregulado, para el desarrollo de las habilidades del pensamiento crítico en el área de educación artística en la disciplina de artes plásticas y visuales y, por último, 3) analizar los factores de la autorregulación del aprendizaje en el diseño de una estrategia didáctica para el desarrollo de las habilidades del pensamiento en el área de educación artística en la disciplina de artes plásticas y visuales.

\section{Marco teórico}

\section{Aprendizaje autorregulado}

La autorregulación según Zimmerman (2000), es un proceso que integra los pensamientos autogenerados, las emociones y todas las acciones que conllevan al logro de las metas ya sea porque se hagan de manera planificada o porque se generen unas nuevas. Siendo así y respetando este principio, la autorregulación del aprendizaje se refiere a aquellas estrategias que genera el aprendiz para alcanzar los objetivos que se proponen. Para Gaxiola y González (2017), la autorregulación es una herramienta importante para el éxito escolar; toda vez que el aprendizaje 
regulado trae consigo factores protectores que coadyuvan al fortalecimiento de este como el apoyo de la familia y de grupos de trabajo, las metas, la disposición del estudiante e incluso la forma en que este afronta las dificultades que se le presentan. Lo que se ratifica por al manifestar que el aprendizaje autorregulado es "un proceso activo en el que el discente selecciona las metas académicas que desea lograr y regula las variables cognitivas, afectivo-motivacionales, contextuales y comportamentales que intervienen en el aprendizaje con el fin de alcanzarlas" (Medina et al., 2019, p. 5).

Como se puede observar la autorregulación es un proceso cíclico que vuelve a iniciar pero que en cada reinicio mejora gracias a los diferentes componentes que explora el estudiante. Cabe anotar, que en cada estudiante el proceso de autorregulación está mediado por las especificidades del sujeto y que estas pueden afectar de manera positiva o negativa la realización de la tarea; sin embargo, cuando el estudiante empieza a dominar las fases de la autorregulación las falencias desaparecen de manera paulatina y se instauran desde la introyección nuevas formas de realización de la tarea y del logro de metas.

Lo anterior presupone, que el aprendizaje autorregulado en el área de artística permitirá que el estudiante no solo aprenda los contenidos, sino que se conozca a sí mismo en el desarrollo de actividades académicas y que lo tome como un hábito en todas las áreas del conocimiento potencializando su aprendizaje y permitiéndole tener éxitos académicos basados en la regulación de su propio aprendizaje.

\section{Pensamiento crítico}

El pensamiento crítico es una construcción que conduce al crecimiento, eficacia y creatividad del pensamiento. Este, es el proceso de analizar y evaluar el pensamiento con el propósito de mejorarlo y presupone el conocimiento de las estructuras más básicas (los elementos del pensamiento) y los estándares intelectuales más básicos del mismo (Paul y Elder, 2005). El pensamiento crítico es reconocido como competencia académica básica aplicable en diversos ámbitos de la vida de cualquier persona, así como la lectura y la escritura, por ello, necesita ser tenido en cuenta en el currículo de toda institución educativa, es decir, se convierte en una habilidad necesaria a desarrollar en las escuelas para garantizar el desarrollo y adaptación del individuo en un mundo en permanente cambio (Causado et al., 2015).

Para algunos autores la competencia del pensamiento crítico, es el pensar claro y racional que favorece el desarrollo de pensamiento reflexivo e independiente, permite a toda persona realizar juicios confiables sobre la credibilidad de una afirmación o la conveniencia de una determinada acción (Guerrero et al., 2018). Por otro lado, el pensamiento crítico, es consustancial a la naturaleza humana. Todo ser humano cuenta con la característica del pensamiento crítico, el cual posee una combinación compleja de habilidades intelectuales empleadas con fines determinados, entre ellos el de analizar cuidadosa y lógicamente información para determinar su validez, la veracidad de su argumentación o premisas y la solución de una problemática (Campos, 2007).

Siendo así, el pensamiento crítico en el aula de educación artística exige pensar acerca de lo que se enseña y lo que se aprende, que el estudiante se cuestione su propia manera de pensar y 
Estrategia didáctica mediada por el aprendizaje autorregulado para el desarrollo del pensamiento crítico en educación artística

se traten de asimilar ideas nuevas y diferentes, haciendo que el alumno regule su pensamiento (autorregulación del aprendizaje), permitiendo que el estudiante incorpore a sus compañeros en su aprendizaje a través de la escucha activa, facilitando la aplicación de lo que se aprende, la comparación de las propias ideas con otras o con lo que el profesor dice, posibilitando que el estudiante explique a otros lo que aprendió y la importancia de ello. El pensamiento crítico proporciona al estudiante habilidades que le permitirán seguir aprendiendo por sí mismo y lidiar positivamente con cualquier información o eventos nuevos facilitando la toma de decisiones.

\section{Estrategia didáctica}

Para el desarrollo de la investigación se tiene en cuenta que una estrategia didáctica es la guía de acciones que se proponen en determinada materia. Esta contiene los pasos, los recursos y la explicación de actividades de aula para los estudiantes (Hérnández et al., 2015). Díaz (1998), define la estrategia didáctica como las instrucciones intencionadas y consientes utilizados para promover y promocionar el aprendizaje significativo. Y Tébar (2003), afirma que son los materiales que utiliza un docente de forma reflexiva para que los estudiantes puedan cumplir los logros de la materia. Otro aspecto importante de las estrategias didácticas es que deben contener la información suficiente (contenidos y material educativo), para que el estudiante aborde las actividades y de esta surge una mayor interacción entre el docente y el estudiante. Siendo el docente un tutor o guía en el conocimiento y no un dador en su totalidad pues facilita espacios de autoaprendizaje y motivación para ello en los estudiantes.

Siendo así, el docente frente al diseño e implementación de una estrategia didáctica debe tener en cuenta unos elementos claves para que dentro de su desarrollo pueda llevar el contenido de manera acertada a la diversidad de estudiantes y de formas de aprender que tiene en el aula de clase; esto conlleva a entender la estrategia didáctica como un acto educativo y humanizador pues están "conformadas por los procesos afectivos, cognitivos y procedimentales que permiten construir el aprendizaje por parte del estudiante y llevar a cabo la instrucción por parte del docente" (Feo, 2010, p. 220). Siendo así, una estrategia didáctica va más allá de la simple imposición de deberes académicos, estas deben liberar toda la creatividad posible para que el estudiante esté motivado a desarrollarla partiendo de las intencionalidades hacia logros determinados con anterioridad lo que permite tener un control de lo enseñado de parte del docente y de lo aprendido de parte del estudiante y es esta conjunción la que permite que la estrategia didáctica sea exitosa.

El rol del estudiante y del docente en esta estrategia propuesta, trasciende los modelos instrumentalistas de la práctica docente donde este solo era un emisor de información y el estudiante un simple receptor sin opinión o desarrollo de ideas propias. Parte de las nuevas miradas del acto educativo y de las implicaciones que tiene poner en contexto la educación para que responda a una realidad globalizada. Siendo así, el docente se convierte en un tutor y el estudiante en un aprendiz que desarrolla sus capacidades a medida que aprende y se reflexiona desde el contexto educativo y humano.

\section{Marco legal}

\section{Educación artística}

Esta obra se comparte bajo la licencia Creative Common Atribución-No Comercial 4.0 International (CC BY-NC 4.0)

Revista de la Universidad Internacional del Ecuador. URL: https://www.uide.edu.ec/ 
Según artículo el artículo 23 de la Ley General de Educación de Colombia, (Ley 115, 1994), la educación artística hace parte de las áreas obligatorias y fundamentales que deben contemplarse dentro del Proyecto Educativo Institucional (PEI) en la educación básica; sin embargo, no se específica su obligatoriedad dentro de la educación media. A pesar de ello, es importante reconocer los aportes del área en el alcance de algunos de los objetivos específicos trazados en el artículo 30 de la misma ley para este nivel educativo, como lo son: fomentar la investigación en cuanto a la realidad nacional frente a aspectos económicos, naturales, políticos y sociales; buscar soluciones a problemas del entorno; promover la participación y responsabilidad en acciones cívicas y sociales; desarrollar habilidades críticas y reflexivas para la comprensión de las dinámicas sociales con relación a la moral, la ética, la religión y la cultura; entre otros (Ministerio de Educación Nacional de Colombia, Ley general de educación, 1994).

Lo anterior se sustenta en lo señalado por el Ministerio de educación Nacional de Colombia (2010) en el documento $\mathrm{N}^{\circ} 16$ "Orientaciones Pedagógicas para el Área de Educación Artística en Básica y Media", en el que se plantea cómo el área posibilita la reconstrucción y apropiación sociocultural en las comunidades mediante acciones como: la conservación de conocimientos y prácticas patrimoniales; la relación estética con el entorno y el afianzamiento del pensamiento reflexivo y crítico frente al mismo; el fortalecimiento de competencias comunicativas, matemáticas, científicas y ciudadanas y el desarrollo de la sensibilidad, la creación y expresión simbólica; lo que a su vez genera el ejercicio de una ciudadanía más democrática y participativa.

Cabe señalar que dentro de los lineamientos curriculares para el área de educación artística propuestos por el Ministerio de Educación Nacional de Colombia (1997), esta se puede orientar desde diferentes campos de forma interdisciplinar; como son: las artes plásticas y visuales, el diseño gráfico, el arte audiovisual, la música, la danza, el teatro, la literatura y la arquitectura. Sin embargo, considerando el contexto global actual, en el que, como lo señala Acaso (2009), existe un hiperdesarrollo del lenguaje visual y del consumo, es preciso ahondar en una educación artística que le permita al estudiante desarrollar habilidades de observación, análisis y decodificación simbólica de la información visual con la que es bombardeado a diario.

\section{Metodología}

El desarrollo de la investigación parte de un enfoque mixto. Siendo así, se toman el enfoque cualitativo y cuantitativo para el estudio. Según Hernández-Sampieri y Mendoza, (2018), los métodos de investigación que utilizan fuentes de información cualitativas y cuantitativas permiten tener información más amplia del fenómeno abordado y el análisis resulta de la triangulación de los hallazgos conservando la estructura de ambos.

El tipo de investigación, es aplicada que según Vargas (2009), permite que el investigador conozca la realidad a través de la evidencia científica. Esta se caracteriza por analizar la realidad y a través de este análisis proponer posibles mejoras a la misma. Desde la investigación aplicada es posible innovar, explorar técnicas diferentes y desarrollar nuevas formas de abordar las problemáticas estudiadas. Partiendo de esta premisa, la investigación aplicada permite utilizar los conocimientos adquiridos desde la teoría y llevarlos a la práctica, poniéndolos al servicio de los participantes del estudio y de la comunidad en general. Igualmente, se adquieren nuevos conocimientos desde los hallazgos del estudio. 
Para el análisis de los datos cuantitativos se eligió un diseño metodológico no experimental, ya que, como lo menciona Hernández-Sampieri y Mendoza (2018) posibilita el estudio de los datos obtenidos partiendo de lo que se observa del fenómeno en sí, tal cual se da en su contexto original, sin identificar la relación existente entre las variables. El diseño seleccionado para el análisis de los datos cualitativos del presente estudio fue el fenomenológico que analiza la realidad entendiéndola en su complejidad y respetando aquellos conocimientos que se encuentran en medio de la investigación al partir de lo que el investigador sabe hacia lo que aprende en el proceso investigativo dando como resultado una trasformación de la realidad abordada (Vargas 2009). El alcance del estudio fue descriptivo, que pretende caracterizar fenómenos, situaciones, eventos indicando sus rasgos más distintivos o diferenciadores. Los estudios descriptivos buscan especificar las propiedades, las características y los perfiles de personas, grupos, comunidades, procesos, objetos o cualquier otro fenómeno que se somete a un análisis. "Es decir, únicamente pretenden medir o recoger información de manera independiente o conjunta sobre los conceptos o las variables a las que se refieren, esto es, su objetivo no es indicar cómo se relacionan estas" (Hernández et al, 2014, p.80).

Para el desarrollo del estudio al ser mixto, se consideró un método inductivo para el estudio cualitativo y un método hipotético deductivo para el estudio cuantitativo. Donde según Cerda (2011), el inductivo parte de fenómenos particulares hacia las descripciones generales del mismo. Esto se refiere a que se parte de la observación y la experimentación de las particularidades del fenómeno hacia los postulados de nivel general. Asimismo Núñez (2017), menciona que ambos enfoques (cualitativo y cuantitativo), recogen y analizan de manera rigurosa los datos cualitativos y cuantitativos, está basado en preguntas de investigación, integra y relaciona cada componente propuesto para el desarrollo de la investigación. Este método cobró gran importancia en la presente propuesta pues su pluralidad le dio el peso a cada aspecto que se desarrolló antes, durante y después del diseño de la estrategia didáctica.

Los participantes de la investigación fueron 27 estudiantes en la fase cuantitativa y 5 docentes con experiencia en el área de educación artística en la fase cualitativa. Teniendo en cuenta que la investigación se desarrolló en medio de la contingencia sanitaria generada por el COVID19, como criterios de inclusión se consideró que los estudiantes participantes fueran del grado décimo y contaran con un dispositivo con acceso a internet. Como criterios de exclusión, se exceptuaron a aquellos estudiantes que durante este tiempo realizaron su proceso educativo a través de guías físicas.

Para determinar el número de estudiantes que participarían del estudio se aplicó la herramienta de cálculo de proporciones. Según este cálculo, el tamaño muestral de la investigación debía de ser de 34 estudiantes; sin embargo, en este estudio la muestra corresponde a aquellos estudiantes que en medio de la problemática de salud asociada al COVID-19 continuaron su proceso de aprendizaje en modalidad virtual y, además, tuvieron la posibilidad de acceder al encuentro sincrónico que se realizó para la aplicación del cuestionario, lo que redujo el número a 27 estudiantes.

Como técnica de recolección de datos para la fase cuantitativa, se seleccionó la encuesta y se aplicó como instrumento un cuestionario a través de la plataforma virtual Google forms, la cual 
arroja los resultados estadísticos de forma sincrónica a su trámite. Para la construcción del mismo se tuvo en cuenta la siguiente operacionalización de variables:

Tabla 1

Matriz de operacionalización de la variable aprendizaje autorregulado

\begin{tabular}{|c|c|c|c|}
\hline Dimensión & Indicadores & Escala de medición & $\begin{array}{l}\text { Niveles y rangos } \\
\text { (valor final) }\end{array}$ \\
\hline Planificación & $\begin{array}{l}\text { Forma en la que se planea el } \\
\text { desarrollo de las actividades } \\
\text { propuestas en el área. }\end{array}$ & $\begin{array}{l}\text { Ordinal } \\
\text { Politómica }\end{array}$ & $\begin{array}{l}\text { 1=Nunca } \\
2=\text { Casi nunca } \\
3=\text { Con frecuencia } \\
4=\text { Siempre }\end{array}$ \\
\hline Ejecución & $\begin{array}{l}\text { Forma en la que desarrolla las } \\
\text { actividades. }\end{array}$ & $\begin{array}{l}\text { Ordinal } \\
\text { Politómica }\end{array}$ & $\begin{array}{l}1=\text { Nunca } \\
2=\text { Casi nunca } \\
3=\text { Con frecuencia } \\
4=\text { Siempre }\end{array}$ \\
\hline Autorreflexión & $\begin{array}{l}\text { Autoevaluación de lo } \\
\text { realizado. }\end{array}$ & $\begin{array}{l}\text { Ordinal } \\
\text { Politómica }\end{array}$ & $\begin{array}{l}1=\text { Nunca } \\
2=\text { Casi nunca } \\
3=\text { Con frecuencia } \\
4=\text { Siempre }\end{array}$ \\
\hline
\end{tabular}

Nota: elaboración propia.

\section{Tabla 2}

Matriz de operacionalización de la variable pensamiento crítico

\begin{tabular}{|c|c|c|c|}
\hline Dimensión & Indicadores & Escala de medición & $\begin{array}{l}\text { Niveles y rangos } \\
\text { (valor final) }\end{array}$ \\
\hline Lógica & $\begin{array}{l}\text { Cuestionamiento de la } \\
\text { información }\end{array}$ & $\begin{array}{l}\text { Ordinal } \\
\text { Politómica }\end{array}$ & $\begin{array}{l}1=\text { Nunca } \\
2=\text { Casi nunca } \\
3=\text { Con frecuencia } \\
\text { 4=Siempre }\end{array}$ \\
\hline Sustantiva & $\begin{array}{l}\text { Manera en la que el estudiante } \\
\text { entrega la información a la } \\
\text { comunidad que lo rodea }\end{array}$ & $\begin{array}{l}\text { Ordinal } \\
\text { Politómica }\end{array}$ & $\begin{array}{l}1=\text { Nunca } \\
2=\text { Casi nunca } \\
3=\text { Con frecuencia } \\
4=\text { Siempre }\end{array}$ \\
\hline Dialógica & $\begin{array}{l}\text { Forma en la que el estudiante se } \\
\text { relaciona en su comunidad }\end{array}$ & $\begin{array}{l}\text { Ordinal } \\
\text { Politómica }\end{array}$ & $\begin{array}{l}1=\text { Nunca } \\
2=\text { Casi nunca } \\
3=\text { Con frecuencia } \\
4=\text { Siempre }\end{array}$ \\
\hline Contextual & $\begin{array}{l}\text { Entendimiento de la realidad en } \\
\text { la que habita }\end{array}$ & $\begin{array}{l}\text { Ordinal } \\
\text { Politómica }\end{array}$ & $\begin{array}{l}1=\text { Nunca } \\
2=\text { Casi nunca } \\
3=\text { Con frecuencia } \\
4=\text { Siempre }\end{array}$ \\
\hline Pragmática & $\begin{array}{l}\text { Autoevaluación de acciones y } \\
\text { posturas propias }\end{array}$ & $\begin{array}{l}\text { Ordinal } \\
\text { Politómica }\end{array}$ & $\begin{array}{l}1=\text { Nunca } \\
2=\text { Casi nunca } \\
3=\text { Con frecuencia } \\
4=\text { Siempre }\end{array}$ \\
\hline
\end{tabular}

Nota: elaboración propia. 
Estrategia didáctica mediada por el aprendizaje autorregulado para el desarrollo del pensamiento crítico en educación artística

Frente del análisis inferencial, el cual está relacionado con la comprobación de la hipótesis, se aplicó una prueba de normalidad mediante el software SPSS, basándose en las pruebas de normalidad postuladas por Shapiro Wilk y Kolgomorov, partiendo de los criterios de decisión en los que: por un parte, si $\mathrm{P}<$ a 0,5 se rechaza la hipótesis $\left(\mathrm{H}_{0}\right)$ y se acepta la hipótesis alterna $\left(\mathrm{H}_{\mathrm{a}}\right)$. $\mathrm{Y}$, por otra parte, si $\mathrm{P}>$ a 0,5 se acepta la hipótesis $\mathrm{H}_{0}$ y res rechaza la hipótesis $\mathrm{H}_{\mathrm{a}}$.

Para la fase de análisis de datos cualitativos, se eligió como técnica de recolección de datos la entrevista y se aplicó como instrumento una guía de entrevista semiestructura basada en la siguiente tabla de matriz categorial:

\section{Tabla 3}

Matriz de categorización para la categoría estrategia didáctica

\begin{tabular}{ll}
\multicolumn{1}{c}{ Subcategorías } & \multicolumn{1}{c}{ Dominios } \\
\hline $\begin{array}{l}\text { Contexto } \\
\text { Recursos y medios }\end{array}$ & Ambientes de aprendizaje. \\
& Material educativo. \\
& Estilos de aprendizaje (estilo icónico, estilo auditivo, estilo \\
kinestésico). & Desarrollo de las competencias específicas del área de educación \\
Competencias & artística. \\
Secuencia didáctica & Rol del docente y del estudiante. \\
Aprendizaje autorregulado & Factores de la autorregulación del aprendizaje. \\
Pensamiento crítico & Actividades de aprendizaje. \\
\hline
\end{tabular}

Nota: elaboración propia.

\section{Resultados y Discusión}

La investigación tuvo como objetivo el diseñar una estrategia didáctica mediada por el aprendizaje autorregulado para el desarrollo de habilidades del pensamiento crítico en el área de educación artística en la disciplina de artes plásticas y visuales en estudiantes de grado décimo de la Institución Educativa Alberto Lebrún Múnera. Para ello, se identificaron las habilidades de pensamiento crítico y las competencias de autorregulación de los estudiantes a través de un cuestionario aplicado en un formulario de Google Drive. Por otro lado, se determinaron los componentes de la estrategia didáctica mediada por el aprendizaje autorregulado, para el desarrollo de las habilidades del pensamiento crítico en el área de educación artística en la disciplina de artes plásticas y visuales a través de una entrevista semiestructurada y partiendo de lo encontrado se diseña una estrategia didáctica mediada por el aprendizaje autorregulado para el desarrollo de habilidades del pensamiento crítico en el área de educación artística en la disciplina de artes plásticas y visuales.

Objetivo específico 1: Identificar las habilidades de pensamiento crítico y las competencias de autorregulación que tienen los estudiantes del grado décimo de la Institución Educativa Alberto Lebrún Múnera.

A continuación, se muestran los resultados con relación a las dimensiones propias de la variable pensamiento crítico partiendo de lo señalado en la figura 1, la cual expone la sumatoria 
de los porcentajes obtenidos en la aplicación del cuestionario frente a los rangos de valor "con frecuencia" y "siempre".

\section{Figura 1}

\section{Habilidades del pensamiento crítico de los estudiantes}

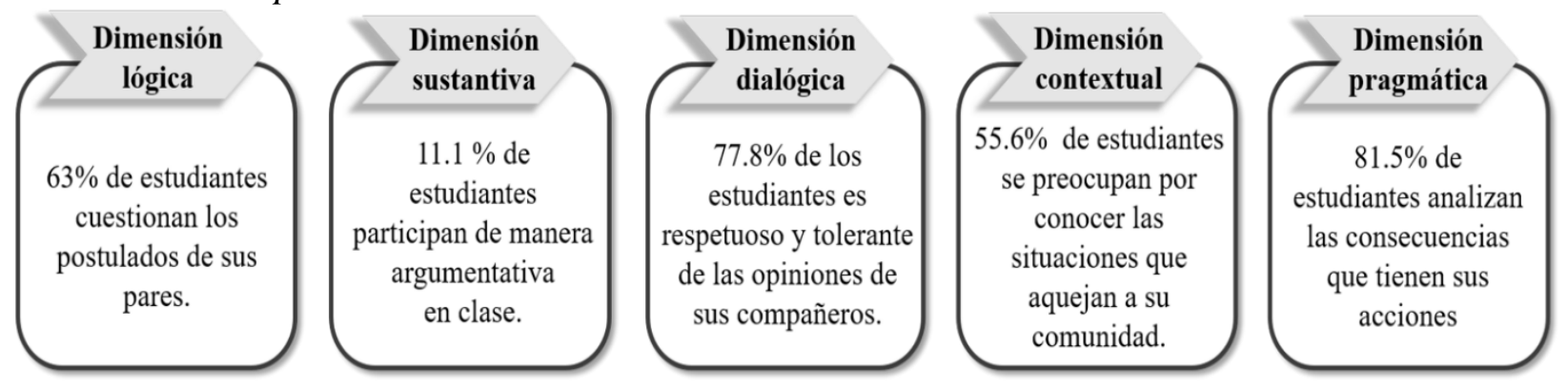

Nota: elaboración propia con base en (Villarini, 2003).

La dimensión lógica, entendiéndola como la capacidad de razonar de manera crítica y lógica los postulados de otro cuando son expuestos de alguna manera en el transcurso de la clase, muestra un resultado optimista, pues la cantidad de estudiantes que cuentan con habilidades con relación a esta es mayoritaria, lo que indica que puede potencializarse en todos los estudiantes en general, aunque teniendo una profundización con aquellos que no exploran esta alternativa de manera frecuente. Tal y como lo expresa Montoya (2007), esta dimensión tiene una estructura determinada por un pensamiento coherente, organizado y sistemático lo que le permite al estudiante pensar de manera más clara y obtener mejores resultados en su proceso de aprendizaje.

En la dimensión sustantiva se encontró que la mayoría de los estudiantes no participan de manera argumentativa en clase, a pesar de tener la habilidad de comprender criterios de manera lógica como se observó en la dimensión anterior. De ahí, se entiende la importancia de realizar actividades que desarrollen esta dimensión pues desde esta se evalúan los postulados de las demás personas haciendo el pensamiento más efectivo para el procesamiento de la información y la comparación de los argumentos propios y los de los demás de manera informada (Montoya, 2007).

En la dimensión dialógica un alto porcentaje de estudiantes son respetuosos y tolerantes con las opiniones de sus compañeros, lo que resulta siendo un aporte importante para la sana convivencia dentro del aula de clase, y su vez en otros entornos sociales. Además, en esta el estudiante fundamenta su pensamiento de manera integral abarcando diferentes puntos de vista para formular el propio, facilitando así la interacción social (Montoya, 2007).

En la dimensión contextual se encuentra que, apenas la mitad de los estudiantes, aproximadamente, se preocupan por conocer las situaciones que aquejan la comunidad en la que viven; por ende, es necesarios fomentar el desarrollo de las habilidades relacionadas con esta dimensión de forma general, pues la existencia de las mismas no es determinante. Es este caso es importante que se realicen actividades encaminadas a que el estudiante entienda y lea los contextos de comunidad y de país con el fin de elevar criterios propios de la situación que se vive en el país y que afecta a las diferentes poblaciones. Esta dimensión es importante en la medida que se 
Estrategia didáctica mediada por el aprendizaje autorregulado para el desarrollo del pensamiento crítico en educación artística

entiende el contexto y el porqué de las cosas que pasan en este partiendo de comprensiones hacia ideologías políticas, reconocimiento de la cultura y de los avances o retrocesos que en esta se ven proponiendo alternativas de solución que parten de dichos análisis (Montoya, 2007).

En la dimensión pragmática se observa que la mayoría de los estudiantes analizan las consecuencias que tienen sus acciones. Esto favorece los ejercicios de autoevaluación que hacen parte de la evaluación formativa y facilitan la mejora de los procesos educativos. Por lo anterior, es pertinente continuar fortaleciendo dentro del aula de clase aquellas habilidades que componen esta dimensión, pues le permite al estudiante generar juicios y mejorar las falencias que se analice y encuentre no tan adecuadas o que tengan consecuencias negativas para su vida y la de los demás. Tal y como lo expresa Montoya (2007), esta dimensión es importante para el pensamiento crítico pues permite examinar y fortalecer a profundidad el pensamiento en la medida en que trasciende los intereses particulares y propicia la madurez intelectual.

Así mismo, se evidencian ahora los resultados con relación a las dimensiones propias de la variable aprendizaje autorregulado, siguiendo lo afirmado en la figura 2 que, como en el caso de la variable anterior, señala la sumatoria de los porcentajes obtenidos en la aplicación del cuestionario frente a los rangos de valor "con frecuencia" y "siempre".

\section{Figura 2}

Competencias de aprendizaje autorregulado de los estudiantes
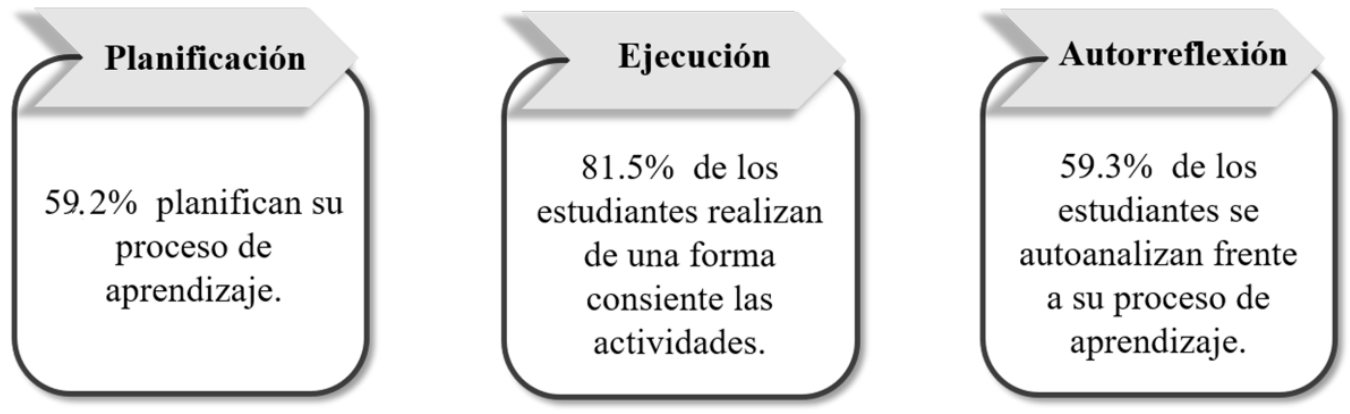

Nota: elaboración propia desde los preceptos de (Zimmerman, 2000).

En la dimensión de planificación se observa que la cantidad de estudiantes que proyectan sistemáticamente su proceso de aprendizaje es predominante; sin embargo, los porcentajes entre los estudiantes que lo hacen y los que no, distan de forma poco significativa, por lo que es fundamental propender por la aplicación de estrategias que les permitan visualizar de manera oportuna cada una de las acciones necesarias para el óptimo desarrollo de sus actividades académicas. Esta competencia es importante trabajarla en el aula de clase para que los estudiantes desarrollen métodos de estudio para tener más éxito a nivel académico desde un aprendizaje autorregulado (Casado et al., 2015).

En la dimensión de ejecución, se obtuvo un resultado favorecedor; sin embargo, considerando de esta se encuentra ligada directamente a la dimensión anterior, pues tiene relación 
con la ejecución de lo planeado previamente para el logro de las metas de aprendizaje, que es necesario fortalecer esta competencia, con el fin de que los estudiantes tengan la posibilidad de realizar los ajustes necesarios para llevar a buen término las actividades de aprendizaje, esto a todas luces permite que el estudiante corrija a partir del análisis reflexivo de lo que se está realizando (Casado et al., 2015).

Los resultados de la dimensión de autorreflexión son similares a los de la dimensión de planificación, lo que quiere decir que gran parte de los estudiantes no se ocupan de autoanalizarse frente a su proceso de aprendizaje. Por ello, se considera que, al ser un porcentaje tan alto, deben realizarse actividades que permitan la aplicación de herramientas de autoevaluación que mejoren los procesos en que el estudiante aborda sus actividades de clase y su aprendizaje, determinando de manera cara que espera aprender y cómo espera hacerlo. Es decir, estrategias que le permitan al estudiante "llevar a cabo procesos de autoevaluación y coevaluación como situaciones generadoras de aprendizaje y donde los instrumentos de evaluación adoptan su carácter verdaderamente formativo" (Casado et al., 2015, p. 215).

Por otro lado, en cuanto al análisis inferencial, de acuerdo con los datos obtenidos en la prueba de normalidad aplicada y descrita en la parte metodológica, $\mathrm{p}$ valor $>0,05$, como se muestra en la tabla 4, lo que llevó a aceptar la hipótesis $\mathrm{H}_{0}$ y se rechazar la hipótesis $\mathrm{H}_{\mathrm{a}}$. La aceptación de la hipótesis $\mathrm{H}_{0}$, la cual plantea que no se identifican habilidades de pensamiento crítico y autorregulación del aprendizaje en los estudiantes que favorezcan el desarrollo de la estrategia didáctica, concuerda con lo encontrado en el análisis cuantitativo, puesto que, teniendo en cuenta los porcentajes arrojados por los rangos de valor "con frecuencia" y "siempre" en la aplicación del cuestionario, si bien algunos estudiantes cuentan con habilidades del pensamiento crítico y competencias de autorregulación del aprendizaje, en general estas deber ser fortalecidas, especialmente las habilidades del pensamiento crítico relacionadas con las dimensiones lógica y contextual y las competencias de autorregulación del aprendizaje referentes a las fase de planificación y autorreflexión.

\section{Tabla 4}

\section{Pruebas de normalidad}

\begin{tabular}{llllllll}
\hline \multicolumn{7}{c}{ Pruebas de normalidad } \\
\hline \multicolumn{7}{c}{ Kolmogorov-Smirnov $^{\mathrm{a}}$} & \multicolumn{3}{l}{ Shapiro-Wilk } \\
\cline { 2 - 7 } Aprendizaje_autorregulado & Estadístico & gl & Sig. & Estadístico & gl & Sig. \\
Pensamiento_crítico & 151 & 27 &, 117 &, 961 & 27 &, 385 \\
& 108 & 27 &, $200^{*}$ &, 958 & 27 &, 341 \\
*. Esto es un límite inferior de la significación verdadera. \\
$\begin{array}{l}\text { a. Corrección de significación de Lilliefors } \\
\text { Los datos no tienen una distribución normal, }\end{array}$ \\
\hline
\end{tabular}

Nota: elaboración desde el software estadístico SPSS. 
Estrategia didáctica mediada por el aprendizaje autorregulado para el desarrollo del pensamiento crítico en educación artística

Objetivo específico 2: Determinar los componentes de la estrategia didáctica mediada por el aprendizaje autorregulado, para el desarrollo de las habilidades del pensamiento crítico en el área de educación artística en la disciplina de artes plásticas y visuales en los estudiantes del grado décimo de la Institución Educativa Alberto Lebrún Múnera. Los componentes de la estrategia pedagógica que surge de las recomendaciones de los docentes.

A continuación, se sintetiza lo analizado en la fase cuantitativa con el apoyo de lo contenido en la figura 3, en la que se delimitan las subcategorías derivadas de la categoría estrategia didáctica, y desde las cuales se trazan los componentes que, según los participantes de la entrevista, debe contener la estrategia.

\section{Figura 3}

Componentes de la estrategia didáctica según la entrevista aplicada a los docentes

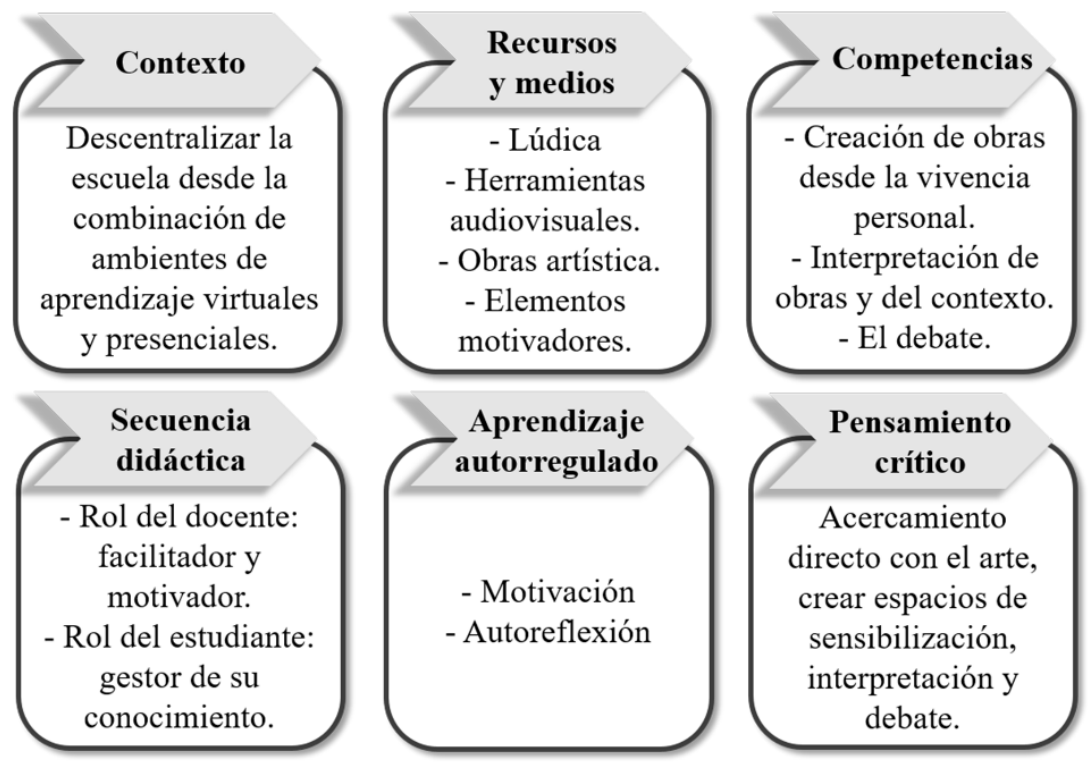

Nota: elaboración propia.

Con relación al contexto, se encuentra que los docentes al enfrentar el cambio de las clases presenciales a la virtualidad por el contexto pandémico que se vive en el mundo, han tenido que reinventarse y encontrar herramientas para llevar el proceso de enseñanza- aprendizaje a los estudiantes.

(...) mire, ahora por el asunto de pandemia nos llevó a descubrir que hay una cantidad de herramientas (...) Yo creo que las herramientas tecnológicas nos pueden ayudar, pero habría que combinar las dos didácticas dándoles (...) la posibilidad a chicos de experimentación, de creatividad, de desarrollar la intuición, la creatividad, el trabajo colaborativo y la resolución de diferentes problemas que se les pueden presentar en el área. (...) pero se podrían que 
combinar las dos metodologías, sin dejar de lado en el momento en que volvamos a la presencialidad la virtualidad. (Entrevistado 1. Línea 57-65).

Siendo así, el docente en la actualidad entiende los nuevos contextos en los que se desarrolla lo educativo. Cabe anotar, que si bien la situación presente del aislamiento y no presencialidad de las clases los docentes reconocen que se debe alternar ambos contextos para que se potencialice la educación. Tal y como manifiesta Duarte (2003), los Ambientes Virtuales de Aprendizaje (AVA), permiten que los estudiantes estén en un espacio que les es familiar por su interacción con las redes sociales lo que permite que se generen interacciones positivas en pro del desarrollo de las temáticas del aula de clase.

En cuanto a los recursos y medios, en general los docentes plantean el usos de herramientas motivacionales como elemento indispensable de las estrategias didácticas, Anaya-Durand y Anaya-Huertas (2010) postulan que se debe educar para aprender y por ello la motivación es vital para desarrollar las diferentes actividades de clase y que además se generen aprendizajes significativos. Tal y como lo expone el Entrevistado 1 para que el estudiante aprenda "debe tener motivación, debe tener emoción, debe utilizar, obviamente, la memoria, pero sin emociones y sin motivación es imposible poder desarrollar el aprendizaje, entonces, obviamente, esta motivación y esta emoción, (...) les permiten la concentración" (línea 74-75). Es decir, la motivación en este caso es el eje del aprendizaje y es necesario entenderlo como una herramienta para desarrollar los diferentes logros de la materia.

Se plantean como competencias a desarrollar: la sensibilidad, la apreciación estética y la comunicación y frente a este ítem los docentes manifiestan que las actividades que se pueden incluir en el diseño de la estrategia didáctica son: creación de obras personales que logren impactar a las demás personas. Según Palet (2018), la educación artística en la escuela busca no formar artistas sino brindar experiencias que lo acerquen a la realidad y al entendimiento de sus contextos y que desde ahí se desarrolle en pensamiento crítico y pueda ejercer su ciudadanía y participar desde los análisis que surgen en los momentos de reflexión que lo circunden.

Tal y como lo expresa el Entrevistado 2 es importante para esta apropiación que sea "algo experimental. Que esa experimentación de lo que ellos vayan a mostrar toque fibras, (...), temáticas personales, pero muy abstractas, (...) pero que cuando una persona lo vea logre transmitir como una sensibilidad y active como ese nivel sensorial" (Línea 66-69), lo que es apoyado por el Entrevistado 3 cuando expresa que para el desarrollo de las competencias específicas del área de artística es importante “(...), la creación de obras muy personales, desde la vivencia de cada estudiante, de acuerdo con una temática específica, posibilita esas tres competencias y la sensibilidad hacia el arte, entender que cada uno tiene una manera de interpretar diferente" (Línea 73-81).

Con respecto a la secuencia didáctica, por un lado, el rol de los estudiantes se percibe desde los entrevistados como dinámico, gestor de su propio conocimiento y motivado. Desde la identificación que el estudiante realiza de sus gustos y este motivado a la tarea puede ayudar a mejorar el proceso de aprendizaje. Es decir, el estudiante debe ser "propositivo, que no se quede solo con la información que vos le das, sino que te pida ir un poco más allá, que sea crítico, que cuestione, que nos desafie a nosotros como docentes en el asunto de educativo" (Entrevistado 3. 
Estrategia didáctica mediada por el aprendizaje autorregulado para el desarrollo del pensamiento crítico en educación artística

Línea 135-140). Por otro lado, el docente debe trascender los modelos tradicionales hacia una nueva mirada de complementariedad entre el conocimiento de este y el del estudiante, porque en la actualidad el educador " (...) ya no es ese profesor parado en el pedestal, que tiene toda la información y que tiene las verdades, ahora nuestro papel es más mediador, más de guía en ese proceso, posibilitando que el estudiante también encuentre sus verdades" (Entrevistado 3. Línea 123-128).

Objetivo específico 3: Analizar los factores de la autorregulación del aprendizaje en el diseño de una estrategia didáctica para el desarrollo de las habilidades del pensamiento en el área de educación artística en la disciplina de artes plásticas y visuales en los estudiantes del grado décimo de la Institución Educativa Alberto Lebrún Múnera.

Retomando lo indicado en la figura 3, en primera instancia uno de los factores que se deben incluir en el diseño de la estrategia didáctica es la motivación. Dentro de las herramientas motivacionales se recomiendan aquellas que permitan que el estudiante mejore su autoconcepto teniendo imágenes más positivas de ellos mismos “(...) para que tengan una visión de ellos positiva, estas estrategias van a ayudar a que el estudiante sea más crítico porque no va a tener miedo de equivocarse en el momento de participar, va ser un estudiante más propositivo" (Entrevistado 5. Línea 149-159). Tal y como menciona Palomo (2014), el autoconcepto influye de manera positiva en la realización de la tarea aumentando la motivación, el aprendizaje y el trabajo en pro de este. En este caso los docentes al igual que Palomo (2014), recomiendan que se realicen actividades de motivación que partan de los intereses y gustos del estudiante para que se enlacen de manera positiva con la actividad.

El estudiante debe encontrar una identificación con ese trabajo que se está haciendo, el estudiante debe tener muy claras las metas de aprendizaje aparte del docente, mientras el estudiante tenga esa claridad va a tener también motivación frente al trabajo y sobre todo seguridad frente a su proceso (Entrevistado 4. Línea 145-156).

Por otro lado, los docentes manifiestan la importancia de que los estudiantes desarrollen un proceso de autovaloración que incluye las expectativas que estos tienen frente a su aprendizaje y al desarrollo de la actividad. Esto, según Zimmerman (2000) se desarrolla en la fase de planificación y la planificación de las metas de aprendizaje nacen de la motivación y la autovaloración que tiene el estudiante de sí mismo incluyendo las expectativas que se tienen del proceso de aprendizaje y el nivel que se desea alcanzar haciendo la tarea. En este desarrollo, la percepción de la autoeficacia tiene gran peso en la ejecución de la misma.

Por ultimo; se resalta que el pensamiento crítico en la educación es importante en la medida que permite que el estudiante haga lecturas contextuales y analice las realidades y de esos análisis pueda proponer posibles soluciones (Palomo, 2014). Estas reflexiones surgen de espacios de debate e interlocución como manifiesta el Entrevistado 1 es importante:

Darles la posibilidad a los chicos de expresarse (...) a lo mejor no van a tener tantos argumentos inicialmente, pero luego, con el ejercicio de acuerdo a cómo vayan desarrollando, van a adquirir nuevos argumentos para poder

Esta obra se comparte bajo la licencia Creative Common Atribución-No Comercial 4.0 International (CC BY-NC 4.0) Revista de la Universidad Internacional del Ecuador. URL: https://www.uide.edu.ec/ 
tener un pensamiento crítico, para poder definir sus ideas, para poder defender sus ideas y para poder sustentar sus argumentos en bases realmente verdaderas y claras (...), yo creo que hay que crearles esas posibilidades de debate, esas posibilidades de argumentación, esas posibilidades de interpretación así se equivoquen, que ellos tengan todas esas posibilidades para que puedan ir enriqueciendo esos aspectos. (Entrevistado 1. Línea 218229).

\section{Conclusiones}

El objetivo de diseñar una estrategia didáctica mediada por el aprendizaje autorregulado para el desarrollo de habilidades del pensamiento crítico en el área de educación artística en la disciplina de artes plásticas y visuales, permite entender la importancia de los procesos de autorregulación dentro del aula de clases pues las competencias de autorregulación trascienden el espacio académico como se observa en trabajos como el de Vallejo (2017), donde habla de la influencia que tiene la autorregulación para el aprendizaje y la disciplina dentro y fuera del aula de clases; es decir, un estudiante que desarrolle estas competencias puede desarrollar pensamiento crítico, control de emociones y convive mejor en sociedad teniendo en cuenta que la autorregulación fomenta tanto el aprendizaje autónomo como el cooperativo pudiendo incluir además a toda la comunidad educativa (González, 2019).

Como lo establece la ley, el área de educación artística es un área obligatoria; por ello, es importante resignificar su valor dentro de los procesos formativos de los estudiantes, pues en la mayoría de los espacios académicos escolares es relegada como un área de poca importancia, lo cual se evidencia, por ejemplo, en el diseño de las pruebas de estado Saber 11, en las que dicha área ni siquiera es evaluada; se desvirtúan totalmente los aportes del arte al desarrollo personal y académico de los estudiantes, lo que se refuerza en el estudio realizado por Steiner (2016), donde manifiesta que las actividades de pensamiento crítico mediadas por la autorregulación del aprendizaje permiten que el estudiante gestione mejor su tiempo, tenga logros y metas establecidas para el desarrollo de la tarea y se permita reflexionar para mejorar constantemente.

Por tal razón, es de gran importancia el rescate que se realizó en esta investigación a las virtudes del arte y el cómo a través de la autorregulación se desarrollan habilidades del pensamiento crítico, lo que en conjunción llevarán a la transformación personal y social en los jóvenes, particularmente, en los estudiantes de básica media que presentan bajo desempeño académico. Es decir, los hallazgos muestran que el estudiante que adquiera una autorregulación en su aprendizaje tendrá más opciones de éxito académico y esto influirá de manera directa en su cotidianidad y en el rol que asume en su educación y en la sociedad (Martínez, 2016).

Al identificar las habilidades de pensamiento crítico y las competencias de autorregulación de los estudiantes desde la mirada cuantitativa se concluye que si bien la mayoría de los estudiantes tienen en ambas variables buenos resultados, hay una gran porción de estudiantes que no tienen las competencias y habilidades que se requieren tanto en el pensamiento crítico como en la autorregulación del aprendizaje y que por ello deben realizarse dentro de la estrategia didáctica actividades que aborden estas habilidades y competencias para que el estudiante pueda llegar a mejorar en lo académico y en lo social. 
La determinación de los componentes de la estrategia didáctica mediada por el aprendizaje autorregulado, para el desarrollo de las habilidades del pensamiento crítico en el área de educación artística en la disciplina de artes plásticas y visuales desde una mirada cualitativa que recogió la percepción de los docentes, permitió entender la importancia que tiene la identificación de las características de los grupos a los que se les brindan los contenidos del área, los gustos y las potencialidades para el desarrollo de clases más enriquecedoras y motivadoras permitiendo que el estudiante desde sus propios intereses generen aprendizajes y trasciendan lo aprendido hacia lo que vive en su cotidianidad (Álvarez, 2018) y (Martínez, et al., 2015).

El análisis cualitativo de los factores de la autorregulación del aprendizaje en el diseño de una estrategia didáctica para el desarrollo de las habilidades del pensamiento en el área de educación artística en la disciplina de artes plásticas y visuales, supone, que la autorregulación también debe ser vista desde el docente pues el rol de guía conlleva a entender las especificidades de cada sujeto, la proyección de lo que se va a evaluar y las expectativas del cómo se va a enseñar a que el estudiante sea autónomo (Moreno-Pinado y Velázquez, 2017). Esta articulación entre el aprendizaje autorregulado y el pensamiento crítico en el aula de artística le da un nuevo significado al contexto educativo y se espera produzca un aprendizaje auténtico como lo expone Villarini (2003), este es un evento que se traduce en un cambio de pensamiento, y de acción en el sujeto que parte de un proceso sistemático y programado para promover dicho aprendizaje.

Por otra parte, es importante mencionar que dentro de las limitaciones existentes en este proceso de investigación se encuentra la anormalidad académica a la que nos vimos sometidos, tanto docentes como estudiantes, a raíz de la pandemia ocasionada por COVID-19, pues esta provocó la deserción de algunos estudiantes y la intermitencia de otros, lo que a su vez ocasionó una reducción en la muestra del estudio cuantitativo.

Finalmente, como recomendaciones se plantean: 1) a nivel institucional, elevar acciones para identificar las habilidades de pensamiento crítico y las competencias de autorregulación que tienen los estudiantes de todos los grados pues esto permite potencializar desde los grados iniciales y mejorar el desempeño escolar, 2) a nivel territorial, se propone generar espacios de formación a los docentes con relación al fortalecimiento del pensamiento crítico, la autorregulación del aprendizaje y la construcción de estrategias didáctica, pues esto permite la generación de nueva propuesta frente a ello en todas las áreas del conocimiento, y 3) a nivel nacional, se recomienda al Ministerio de Educación Nacional generar acciones para la re-significación del valor del área de educación artística en virtud de sus aportes al desarrollo del proceso como el pensamiento crítico y la trasformación sociocultural.

\section{Referencias Bibliográficas}

Acaso, M. (2009). La educación artística no son manualidades: nuevas prácticas en la enseñanza de las artes y la cultura visual. Catarata.

Álvarez Camacho, J. M. (2018). La autorregulación en el aprendizaje del estudiante durante el proceso artístico de la creación consciente. [Tesis de Maestría en Educación, 
Corporación Universitaria Minuto de Dios, Villavicencio- Meta, Colombia]. https://hdl.handle.net/10656/9992.

Anaya-Durand, A., y Anaya-Huertas, C. (2010). ¿Motivar para aprobar o para aprender? Estrategias de motivación del aprendizaje para los estudiantes. Tecnología, Ciencia, Educación, 25(1), 5-14. https://www.redalyc.org/articulo.oa?id=48215094002

Campos Arenas, A. (2007). Pensamiento crítico. Técnicas para su desarrollo. Libreria de la $U$ contenidos + soluciones. https://bit.ly/2WHGuvD.

Casado, B., O., Casado, B., P., y Pérez, P., A. (2015). Capítulo 12. La autorregulación en educación primaria. Una propuesta para favorecer la autonomía del alumnado. En Evaluación formativa y compartida en educación: Experiencias de éxito en todas las etapas educativas. Relieve. https://tinyurl.com/y3rnu2nc

Causado Escobar, R., Carrasco Santos, B. y Salas Calderón, I. (2015). «Desarrollo del pensamiento crítico en el área de ciencias naturales en una escuela de secundaria». Revista de la Facultad de Ciencias 4(2):17-42. https://doi.org/10.15446/rev.fac.cienc.v4n2.51437.

Cerda, H. (2011). Los elementos de la investigación: Como reconocerlos, diseñarlos y construirlos (Segunda). Cooperativa Editorial Magisterio.

Duarte, J. (2003). Ambientes de aprendizaje. Una aproximación conceptual. Revista Iberoamericana de Educación, 33(1), 1-19. https://doi.org/10.35362/rie3312961

Feo, Ronald. (2010). «Orientaciones básicas para el diseño de estrategias didácticas». Tendencias Pedagógicas (16), 220-36. http://doi.org/10.15366/tp

Gaxiola Romero. J., C., y Lugo González, S. (2017). Apoyo percibido, resiliencia, metas y aprendizaje autorregulado en bachilleres. Revista Electrónica de Investigación Educativaredie 21(8), 1-10. https://doi.org/10.24320/redie.2019.21.e08.1983

Guerrero, H., Polo, S., Martínez Royert, J. y Ariza, P. (2018). Trabajo colaborativo como estrategia didáctica para el desarrollo del pensamiento crítico. 86(2018), 959-986. http://hdl.handle.net/11323/2262

Hernández Arteaga, I., Recalde Meneses, J., y Luna, J. (2015). Estrategia didáctica: una competencia docente en la formación para el mundo laboral. Revista Latinoamericana de Estudios Educativos (Colombia), 11(1),73-94. https://www.redalyc.org/articulo.oa?id=134144226005

Hernández-Sampieri, R. y Mendoza, C (2018). Metodología de la investigación. Las rutas cuantitativa, cualitativa y mixta, Ciudad de México, México: Editorial Mc Graw Hill Education.

Medina-Ramírez, R., Álamo-Arce D., Costa, M. y Rodríguez de Castro, F. (2019). Aprendizaje autorregulado: una estrategia para 'enseñar a aprender' en ciencias de la salud. FEM: Revista de la Fundación Educación Médica, 22(1), 5-10. https://doi.org/10.33588/fem.221.981

Ministerio de Educación Nacional de Colombia. (2010). Orientaciones pedagógicas para la educación artística en la básica y media. Revolución educativa Colombia aprende. https://www.mineducacion.gov.co/1759/articles241907_archivo_pdf_orientaciones_artes.pdf

Ministerio de Educación Nacional de Colombia. (1997). Serie de lineamientos curriculares del área de educación artística. https://www.mineducacion.gov.co/1621/articles339975_recurso_4.pdf. 
Estrategia didáctica mediada por el aprendizaje autorregulado para el desarrollo del pensamiento crítico en educación artística

Montoya, J. (2007). Primer avance de investigación. Acercamiento al desarrollo del pensamiento crítico, un reto para la educación actual. Revista Virtual Universidad Católica del Norte, (21). https://www.redalyc.org/articulo.oa?id=194220390001

Moreno-Pinado, W. y Tejeda Velázquez, M. (2017). Estrategia didáctica para desarrollar el pensamiento crítico. REICE. Revista Iberoamericana sobre Calidad, Eficacia y Cambio en Educación 15(2). https://doi.org/10.15366/reice2017.15.2.003.

Núñez Moscoso, J. (2017). Los métodos mixtos en la investigación en educación: hacia un uso reflexivo. Cuadernos de Pesquisa 47(164), 632-

49. https://doi.org/10.1590/198053143763

Palet, M. (2018). ¿Qué es educación artística? Comprender cuál es el territorio propio de la educación artística. issuu.com. https://bit.ly/3kQbV2d

Palomo del Blanco, M. (2014). El autoconcepto y la motivación escolar: una revisión bibliográfica. International Journal of Developmental and Educational Psychology, 6(1), 221-228. https://www.redalyc.org/articulo.oa?id=349851790026

Panadero, E. y Alonso-Tapia, J. (2014). Teorías de autorregulación educativa: una comparación y reflexión teórica. Psicología Educativa 20(1), 11-22. https://doi.org/10.1016/j.pse.2014.05.002.

Richard, P. y Elder, L. (2005). Una Guía Para los Educadores en los Estándares de Competencia para el Pensamiento Crítico Estándares, Principios, Desempeño Indicadores y Resultados Con una Rúbrica Maestra en el Pensamiento Crítico. Fundación para el pensamiento crítico. https://www.criticalthinking.org/resources/PDF/SP-Comp_Standards.pdf

Sánchez Henao, L. (2017). Desarrollo de habilidades de pensamiento crítico a través del Aprendizaje basado en juegos para la Educación Ambiental en estudiantes del grado 5 de primaria. Tesis de Maestría en Educación Ambiental, Universidad de Ciencias Aplicadas y Ambientales U.D.C.A., Bogotá, Colombia. https://repository.udca.edu.co/handle/11158/890

Steiner Hillary, H. (2016). The Strategy Project: Promoting Self-Regulated Learning through an Authentic Assignment. International Journal of Teaching and Learning in Higher Education, 28, 271-82. https://files.eric.ed.gov/fulltext/EJ1111151.pdf

Tapia Benavides, A., Caicedo Melecio, Y., y Caicedo Contreras, Z. (2017). La lúdica como herramienta para el aprendizaje. [Tesis de Maestría en Educación, Universidad Pontificia Bolivariana, Puerto Asís, Putumayo, Colombia]. http://hdl.handle.net/20.500.11912/3382

Tébar Belmonte, L. (2003). El perfil del profesor mediador: pedagogía de la mediación. Madrid: Santillana.

Tolosa Rivera, A. (2015). El arte como posible herramienta metodológica para la construcción de paz. [Tesis de Especialista en acción sin daño y construcción de paz, Facultad de Ciencias Humanas. Universidad Nacional de Colombia, Bogotá, Colombia]. http://enredars.org/sites/default/files/publicaciones/trabajofinalangelatolosa.pdf

Vallejo, R. (2017). La autorregulación y la disciplina escolar de los estudiantes de primero de bachillerato general unificado de la unidad educativa general Eloy Alfaro delgado, cantón Ambato, provincia Tungurahua [Facultad de Ciencias Humanas y de la Educación Carrera de Psicología Educativa, Universidad Técnica De Ambato]. https://bit.ly/2A2IpTJ 
Vargas Cordero, Z. (2009). La investigación aplicada: una forma de conocer las realidades con evidencia científica. Educación 33(1), 155-65. https://doi.org/10.15517/REVEDU.V33I1.538

Villarini, A. (2003). Teoría y pedagogía del pensamiento crítico. 3-4, 8. http://pepsic.bvsalud.org/pdf/pp/v3-4/v3-4a04.pdf

Zimmerman, B. J. (2000). Chapter 2 - Attaining Self-Regulation: A Social Cognitive Perspective». Pp. 13-39 en Handbook of Self-Regulation, editado por M. Boekaerts, P. R. Pintrich, y M. Zeidner. San Diego: Academic Press. https://doi.org/10.1016/B978$\underline{012109890-2 / 50031-7}$ 